\title{
Full versus decoupled constant matrices to speed up power system state estimation
}

\author{
Meriem Majdoub ${ }^{1}$, Bouchra Cheddadi ${ }^{2}$, Omar Sabri $^{3}$, Abdelaziz Belfqih ${ }^{4}$, Jamal Boukherouaa ${ }^{5}$ \\ 1,4,5 Laboratory of Electric Systems and Energy, ENSEM, University Hassan II of Casablanca, Morocco \\ ${ }^{2,3}$ Laboratory RITM, EST, University Hassan II of Casablanca, Morocco
}

\begin{tabular}{l} 
Article Info \\
\hline Article history: \\
Received Jul 15, 2019 \\
Revised Mar 18, 2020 \\
Accepted Apr 13, 2020 \\
\hline Keywords: \\
Decoupled constant matrices \\
Full constant matrices \\
Power system state estimation \\
Weighted least squares \\
algorithm
\end{tabular}

Article Info

Article history:

Received Jul 15, 2019

Revised Mar 18, 2020

Keywords:

Decoupled constant matrices Full constant matrices Weighted least squares algorithm

\begin{abstract}
This paper presents a performance evaluation of two solutions to reduce computational burden of the traditional Weighted Least Squares Algorithm for power system state estimation: Simplified methods SWLS1 / SWLS2 based on full constant matrices and Fast decoupled FDWLS based on decoupled constant matrices. First, the algorithms were tested on IEEE 14 and 118 bus transmission systems. Second, the solutions were tested on a rural distribution feeder to evaluate the response of the algorithms to high $\mathrm{R} / \mathrm{X}$ ratio. Results show that for transmission systems, FDWLS is the fastest method but more sensitive to erroneous measurements. Simplifications considered in FDWLS, are not valid in distribution systems with high R/X ratio this results in slowing down the algorithm convergence speed considerably compared to SWLS2 which performs well. SWLS2 algorithm presents a promising solution to reduce computation time for application in future smart grid.
\end{abstract}

This is an open access article under the CC BY-SA license.

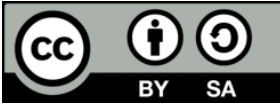

\section{Corresponding Author:}

Meriem Majdoub,

Laboratory of Electric Systems and Energy, ENSEM

ENSEM, University Hassan II of Casablanca,

ENSEM, El Jadida Road, km 7, BP: 8118, Oasis - Casablanca, Morocco.

Email: majdoub.meriem@gmail.com

\section{INTRODUCTION}

Recently, the power system is undergoing big changes due to the massive integration of renewable energy resources to transmission and distribution systems which leads to a complex bidirectional power flow. This conjucture incites researchers to improve the speed and reliability of state estimation algorithms to ensure an efficient real time monitoring of futur Smat Grid.

The weighted least-squares (WLS) method is the most State Estimation Algorithm used in control centers all over the world. In fact, WLS state estimation algorithm provides the best estimation quality and good convergence rate. However, the gain and Jacobian matrices need to be recalculated each iteration which needs a large amount of calculation, a big memory requirement and long computing time [1]. One of the major solutions proposed in literature to circumvent the computational burden is the Fast-decoupled WLS (FDWLS) technique based on decoupled constant matrices [1-5] as used to speed up load flow calculation [6-8]. The fast-decoupled formulation has proven its efficiency to reduce computation time and data storage and has found wide acceptance in the industry, various versions have been implemented in control centers all over the world [4]. However, the decoupled method may fail to provide a solution on illconditioned systems as high $\mathrm{R} / \mathrm{X}$ ratio of distribution branches or in presence of erroneous measurements. Therefore, it will be interesting to develop techniques which ensure a compromise between the reliability of full Weighted Least Squares Algorithm and Convergence speed of fast decoupled WLS and able to overcome 
limitations discussed above. Authors of [5], proposed two variants of WLS method with full constant matrices (SWLS1 and SWLS2) based on Dishonest Gauss Newton Method used for Load Flow [2, 9-11] to reduce calculation time without decoupled simplifications. Though the principle of these methods is very popular, few studies analysed its convergence performances [5, 12-15] on Transmission systems and no study apply it before to a distribution system. This article is the first one exploring the performance of full versus decoupled constant matrices on distribution system state estimation with WLS algorithm. The rest of the paper is organized as follow: Section 2 presents a description of the traditional weighted least squares algorithm and the alternative solutions to reduce computational burden. The algorithms studied are tested both on transmission and distribution systems, employing accurate and erroneous measurements. Simulation results are presented and discussed in Section3. The main findings of the paper are as follows:

- $\quad$ For Transmission Systems, FDWLS based on decoupled constant matrices is the best method, it is 2 to 5 times faster than basic WLS and requires half storage capacity.

- $\quad$ SWLS1 method considering constant Jacobian matrix only is not reliable and does not represents any advantage on reducing computation time.

- $\quad$ For distribution systems, FDWLS based on decoupled constant matrices converges slowly and requires high iterations number while the SWLS2 algorithm evaluating the Jacobian and gain matrices once at the flat start is 2 times faster than basic WLS with the same reliability which make it less sensitive to erroneous measurements and high R/X ratio compared to FDWLS. Therefore, SWLS2 presents a good alternative to reduce computation time in future power systems.

\section{WEIGHTED LEAST SQUARES ALGORITHMS}

\subsection{Basic algorithm}

The Network model employed is the single-phase model with $\mathrm{N}$ buses and $\mathrm{m}$ measurements gathered from remote meters. Most commonly used measurements are the line power flows, bus power injections and bus voltage magnitudes.

The aim of state estimator is to provide the best possible values of the bus voltage magnitudes and angles by processing the available network data recognizing that there are errors in the measured quantities.

The starting equation for the WLS state estimation algorithm is [4, 16-18]:

$z=h(x)+e$

Where: $\mathrm{z}$ is the $(\mathrm{mx} 1)$ measurement vector;

$\mathrm{x}$ is an (nx1) state vector to be estimated: The number of estimated states is $\mathrm{n}=2 * \mathrm{~N}-1$, since the balance phase's is already known $\theta=0$.

$\mathrm{e}$ is an $(\mathrm{mx} 1)$ measurement error vector.

$\mathrm{h}$ is the vector of nonlinear functions that relate the states to the measurements defined:

Real and Reactive power injection at bus i:

$$
\begin{aligned}
& P i=V i \sum_{i \neq j} V j(G i j \cos \theta i j+B i j \sin \theta i j) \\
& Q i=V i \sum_{i \neq j} V j(G i j \sin \theta i j+B i j \cos \theta i j)
\end{aligned}
$$

Real and reactive power flow from bus i to bus $\mathrm{j}$ :

$$
\begin{aligned}
& P i j=V i^{2}(g s i+g i j)-V i V j(g i j \cos \theta i j+b i j \sin \theta i j) \\
& Q i j=-V i^{2}(b s i+b i j)-V i V j(g i j \sin \theta i j-b i j \cos \theta i j)
\end{aligned}
$$

Where:

$V i$ is the voltage magnitude at bus $\mathrm{i}$

$\theta i$ is the phase angle at bus $\mathrm{i}$

$\theta i j=\theta i-\theta j$

$G i j+j B i j$ is the ijth element of the Y-bus matrix

$g i j+j b i j$ is the admittance of the series branch between bus $i$ and bus $j$

$g s i+j b s j$ is the admittance of the shunt branch at bus $\mathrm{i}$.

In practice, it is required to have the number of measurements larger than number of states, this is called redundancy [19]. So, state estimator can consider the various operation layouts used and to cover for the unavailability of transmission and telemetering equipment failures [20]. A measure of the redundancy may be denoted by the redundancy factor $\eta$, which is defined as [21]: 
$\eta=\frac{\text { Dimension of } z}{\text { Dimension of } x}=\frac{m}{n}=\frac{m}{2 N-1}$

The measurement errors ei are assumed to satisfy the following statistical properties:

First, the errors have zero mean: $\mathrm{E}(\mathrm{ei})=0, \mathrm{i}=1, \ldots, \mathrm{m}$. Second, the errors are assumed to be independent, such that the covariance matrix is diagonal:

$\operatorname{Cov}(e)=E\left(e, e^{T}\right)=R=\operatorname{diag}\left\{\sigma_{1}^{2}, \sigma_{2}^{2}, \ldots, \sigma_{m}^{2}\right\}$

The solution to the state estimation problem can be formulated as a minimization of the following objective function:

$$
J(x)=\sum_{i=1}^{m} \frac{\left(z_{i}-h_{i}(x)\right)^{2}}{R_{i i}}=[z-h(x)]^{T} R^{-1}[z-h(x)]
$$

To find the minimization of this objective function the derivative should be set to zero. The derivative of the objective function is denoted by $\mathrm{g}(\mathrm{x})$ :

$$
g(x)=\frac{\partial J(x)}{\partial x}=-H^{T}(x) R^{-1}[z-h(x)]=0
$$

where: $\mathrm{H}(\mathrm{x})=\partial \mathrm{h}(\mathrm{x}) / \partial \mathrm{x}$, called the measurement Jacobian matrix.

Ignoring the higher order terms of the Taylor series expansion of the derivative of the objective functions yields an iterative solution as shown below:

$$
x^{k+1}=x^{k}+\left[G\left(x^{k}\right)\right]^{-1}\left[\left[H\left(x^{k}\right)\right]^{T}[R]^{-1}\left[z-h\left(x^{k}\right)\right]\right]
$$

Where the gain matrix, $\mathrm{G}$, is defined as:

$$
G\left(x^{k}\right)=\frac{\partial g(x)}{\partial x}=H^{T} R^{-1} H
$$

For the first iteration of the optimization problem, an initial guess has to be made for the state vector $\mathrm{x} 0$ corresponding to the flat voltage profile, or flat start. A flat start refers to a state vector where all the voltage magnitudes are 1.0 per unit and all the voltage angles are 0 degrees. With respect to the state vector: the measurement functions, Jacobian and gain matrices are calculated each iteration until the absolute difference between two successive values of $\mathrm{x}$ is less than a chosen tolerance $\varepsilon$. WLS flowchart presented in Figure 1, resumes the principal steps of the algorithm.

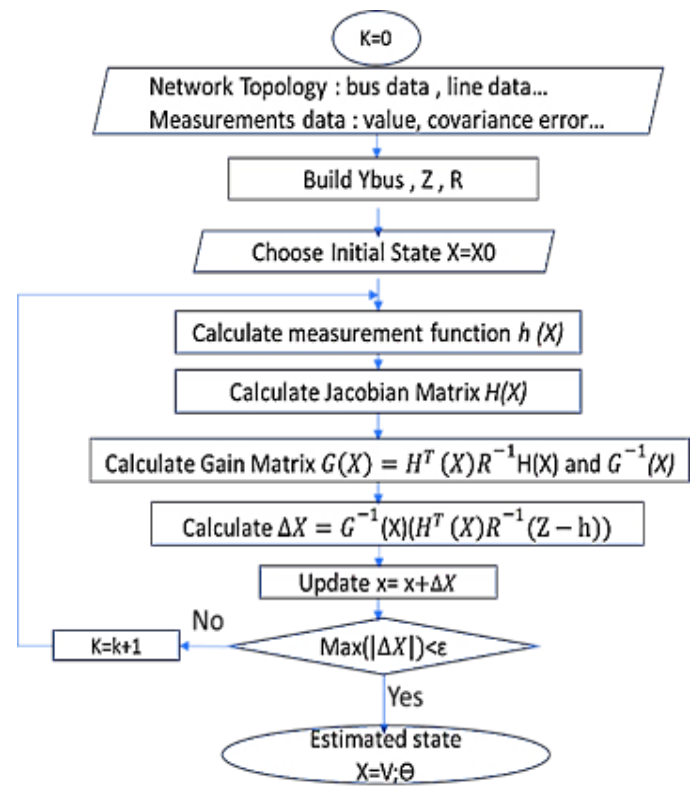

Figure 1. Basic WLS flowchart 


\subsection{Simplified algorithms with full constant matrices}

Mostly, it is observed that the elements of Jacobian and gain matrices do not significantly change between flat start initialization and the converged solution [5]. So, their evaluation could be restrained to some first iterations without altering the estimation quality. Based on this principle two methods have emerged: SWLS1 and SWLS2.

\subsubsection{The first simplified method SWLS1}

The first simplified method (SWLS1) calculates the Jacobian matrix at every iteration but preserves the gain matrix constant after an iteration $\mathrm{k}$ chosen as presented in Figure 2. The obvious advantage of this method is to reduce the number of calculations of the gain matrix.

\subsubsection{The second simplified method SWLS2}

The second method SWLS2 admits that the gain and the Jacobian matrices remain constant after an iteration $\mathrm{k}$ chosen as presented in Figure 3 [5, 13-15].

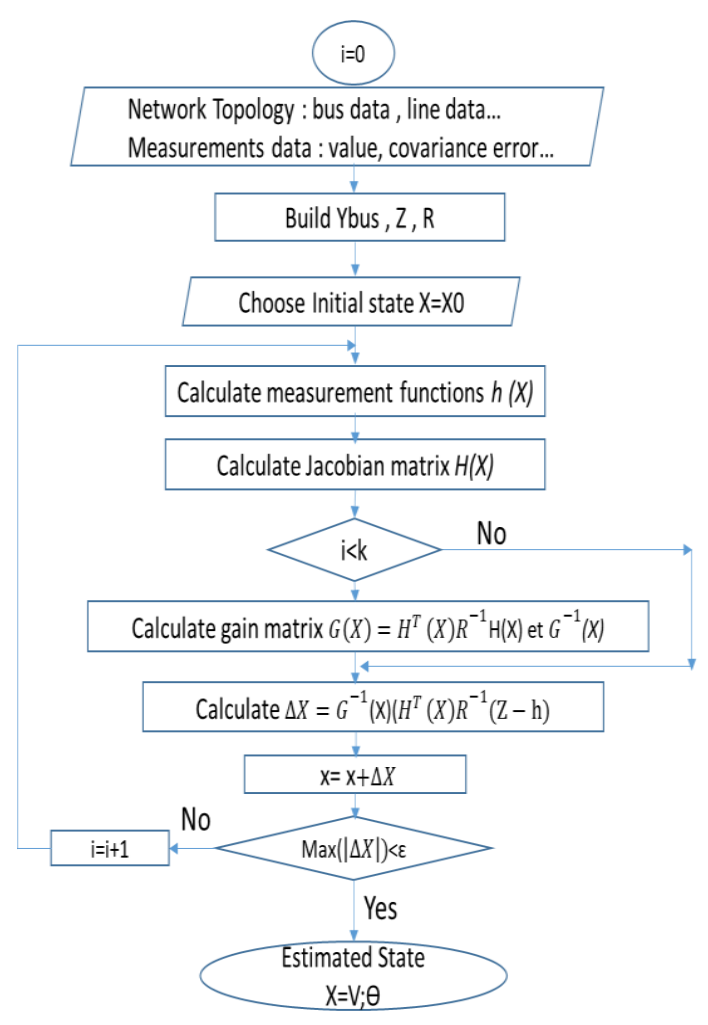

Figure 2. SWLS1 flowchart

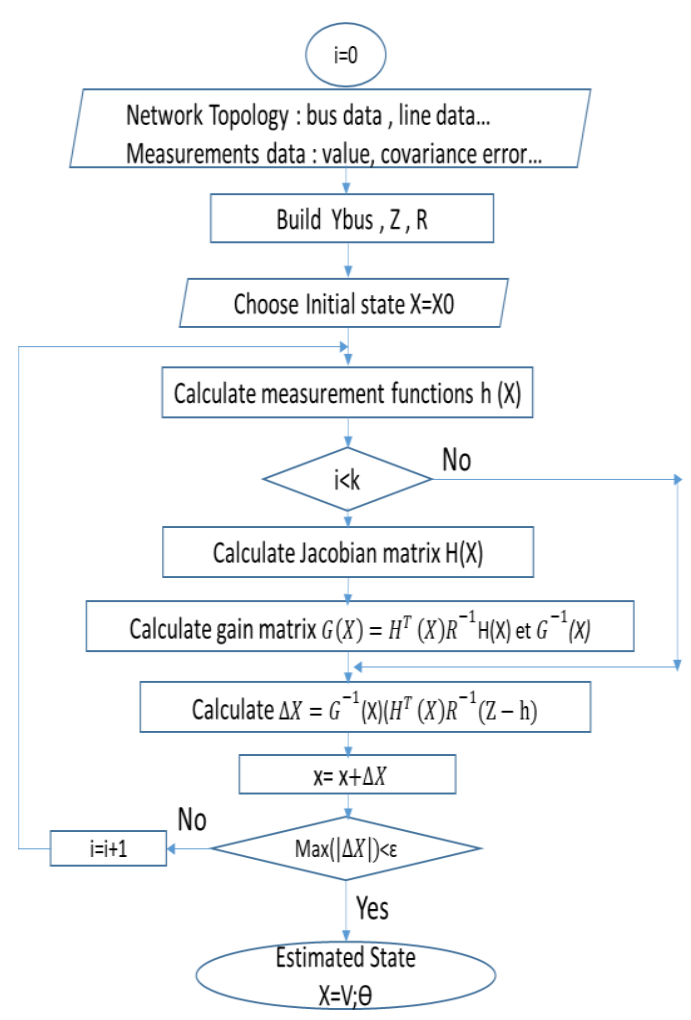

Figure 3. SWLS2 flowchart

\subsection{Fast decoupled weighted least squares algorithm (FDWLS)}

The principle of Fast Decoupled State Estimation Techniques consists on exploiting the active/reactive decoupling property based on fast decoupled load flow methods [2, 9-11]. Indeed, for large scale power systems the transmission lines have a very high $\mathrm{X} / \mathrm{R}$ ratio. In such a case, the real power changes are less sensitive to changes in voltage magnitude and can be ignored. Similarly, the reactive power change is less sensitive to changes in angles. Making these simplifications, the gain matrix in WLS state estimation algorithm can be simplified [10-11].

In the decoupled formulation, the measurement vector $\mathrm{Z}$ is portioned into two parts: active $\mathrm{ZA}$ and reactive ZR components [2-4].

$$
\mathrm{Z}=\left(\begin{array}{c}
Z A \\
Z R
\end{array}\right), \mathrm{Z}_{\mathrm{A}}=\left(\begin{array}{c}
\text { Pinj } \\
\text { Pflow }
\end{array}\right), \mathrm{Z}_{\mathrm{R}}=\left(\begin{array}{c}
\text { Qinj } \\
Q \text { flow } \\
V
\end{array}\right)
$$


Where:

Pinj, Qinj: are respectively real and reactive power injection measurements.

Pflow, Qflow: are respectively real and reactive power flow measurements.

$\mathrm{V}$ : Voltage measurements.

By the definition above the Jacobian matrix $\mathrm{H}$, and covariance matrix $\mathrm{R}$ can be written as:

$\mathrm{H}=\left[\begin{array}{ll}H_{A A} & H_{A R} \\ H_{R A} & H_{R R}\end{array}\right], \mathrm{R}=\left[\begin{array}{cc}R_{A} & 0 \\ 0 & R_{R}\end{array}\right]$

Based on P- $\Theta$ and Q-V decoupling, the off-diagonal blocks $H_{A R}$ and $H_{R A}$ in the measurement jacobian $\mathrm{H}$ are ignored:

$\mathrm{H}=\left[\begin{array}{cc}H_{A A} & 0 \\ 0 & H_{R R}\end{array}\right]$

Then the gain matrix is expressed as follow:

$\mathrm{G}=\left[\begin{array}{cc}G_{A A} & 0 \\ 0 & G_{R R}\end{array}\right]$

Where:

$G_{A A}=H_{A A}^{T} R_{A}^{-1} H_{A A}$

$G_{R R}=H_{R R}^{T} R_{R}^{-1} H_{R R}$

Several versions of the fast-decoupled state estimator have been proposed in literature depending upon the assumptions and approximations adopted. The version recognized as presenting best performance has the following features [2]:

- The Jacobian and gain matrices are evaluated once, at the flat voltage start: all $\mathrm{V}=1$ p.u and $\theta=0$ degrees.

- $\quad$ The branch series resistances are ignored in forming the elements of the Jacobian $\mathrm{H}$, which is equivalent to replacing branch susceptances bij by $-1 / \mathrm{xij}$. Where xij is the reactance of the series branch between bus $\mathrm{i}$ and bus $\mathrm{j}$.

- A transformed measurement vectors $Z A^{\prime}$ and $Z R^{\prime}$ are used by dividing the flow and injection measurements by the corresponding calculated voltage magnitude.

- $\quad$ For better convergence characteristics, a block sequential solution scheme is used wherein:

$G_{A A} \Delta \theta=T A$

$T A=H A A^{T * R A^{-1} * \Delta Z A^{\prime}}$

$\Delta \mathrm{ZA}^{\prime}=(\mathrm{ZA}-\mathrm{hA}) / \mathrm{V}$

$\mathrm{hA}$ is the vector of active measurements functions.

$\Delta \theta$ is solved and the updated $\theta^{\prime} s$ are used in the RHS of:

$G_{R R} \Delta V=T R$

Which is then solved for $\Delta V$.

$T R=H R R^{T * R R^{-1 *} \Delta Z R^{\prime}}$

$\triangle Z R^{\prime}=(Z R-h R) / V$

$\mathrm{hR}$ is the vector of reactive measurements functions.

For more details, about how to calculate the elements of the fast-decoupled Jacobian, reader can refer to [2-4]. Algorithm steps are depicted in Figure 4: 


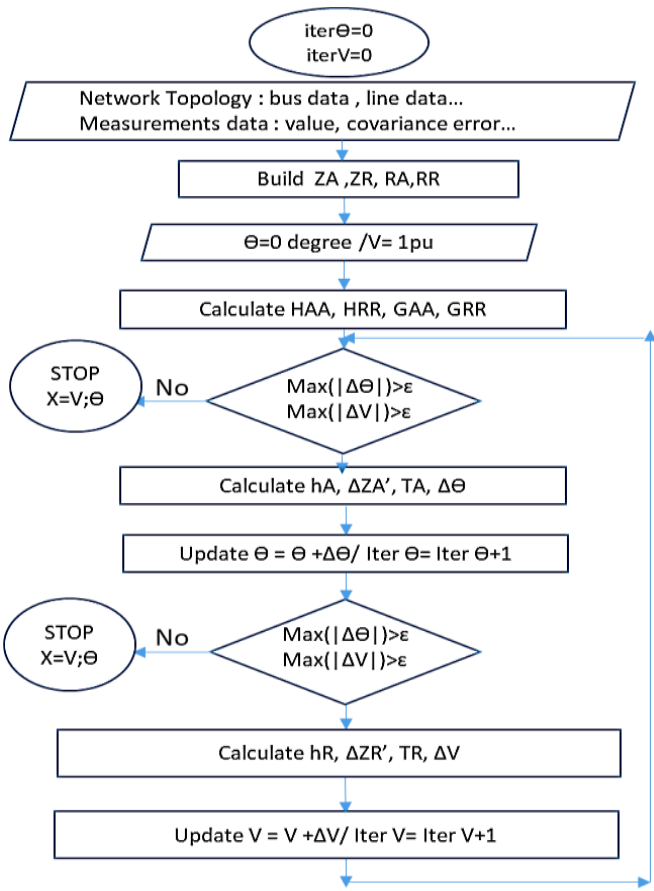

Figure 4. FDWLS flowchart

\section{SIMULATION RESULTS}

The objective of this section is to compare the performance of the Weighted Least Squares Algorithms described in the previous Section: Basic WLS, SWLS1, SWLS2 and FDWLS in terms of computation time, convergence rate, estimation quality and number of iterations.

\subsection{Description of simulations}

The four algorithms have been tested on three study cases IEEE 14, 118 bus systems and a 12-bus rural distribution system under two scenarios: In scenario 1, accurate measurements equal to load flow results have been employed. In scenario 2, measurements were altered by a noise of $10 \%$ to evaluate the response of the studied algorithms to erroneous measurements.

For IEEE bus systems, the network data files can be downloaded from Power Systems Test Case Archive [22].

To compare the state estimate accuracy of the following simulations, mean absolute percentage error (MAPE) is introduced as follow [23]:

$$
M A P E=\frac{1}{n} \sum_{t=1}^{n}\left|\frac{X_{t}-X_{e}}{X_{t}}\right| \times 100 \%
$$

Where, $\mathrm{X} t$ is the true value of system state obtained from load flow results and $\mathrm{Xe}$ is the estimated state. A smaller value of MAPE indicates a more accurate state estimation result.

MAPEV: mean absolute percentage estimation error of voltage magnitude.

MAPEӨ: mean absolute percentage estimation error of voltage angle.

The simplified algorithms have been tested for different value of the iteration $\mathrm{k}$ from which just the gain matrix is considered constant SWLS1 or the gain and Jacobian matrices are constant (SWLS2). Convergence tolerance used in these tests is: $\varepsilon=10-4$ for both voltage magnitude and voltage angle states.

\subsection{Test systems}

For all test cases, measurements were set to ensure a redundancy factor $>1$. They were chosen of different types and uniformly distributed through the network to ensure observability $[16,17]$. Weight of all measurements is assumed 1 .

a. IEEE 14 bus system:

For IEEE 14 bus system test case, a set of 41 measurements $(\eta=1,5)$ is chosen as: 
- 1 voltage magnitude at bus 1 .

- 8 real power injections and 8 reactive power injections at buses: 2, 3, 7, 8, 10, 11, 12, 14 .

- 12 real power flow and 12 reactive power flow on branches: 1-2, 2-3, 4-2, 4-7, 4-9, 5-2, 5-4, 5-6, 6-13, 7-9, 11-6, 12-13.

b. IEEE 118 bus system:

A set of 726 measurements $(\eta=3)$ is chosen a follow:

18 voltage magnitudes for all buses.

- 118 real power injections and 118 reactive power injections for all buses.

- 186 real power flow and 186 reactive power flow for all network branches.

c. 12 bus distribution system:

A physically existing rural distribution Feeder [24] is considered. Figure 5 shows the single-line diagram of the system, where node 1 is the substation. The system data including line data and load data can be found in [25].

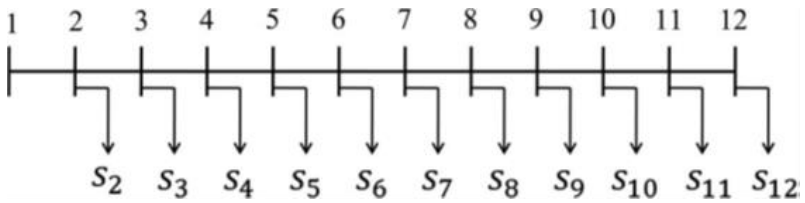

Figure 5. Single-line diagram of the 12-bus distribution system.

A set of 25 measurements $(\eta=1,1)$ is chosen as follow:

- 1 voltage magnitude at bus 1 .

- 1 real power injection and 1 reactive power injection at bus 1 .

- $\quad 11$ real power flow and 11 reactive power flow for all network branches.

\subsection{Results of scenario 1 (accurate measurements)} flow results.

In this section, WLS algorithms have been tested with accurate measurements equal to load

\subsubsection{Simulation results for IEEE 14 bus system}

As seen in Table 1:

FDWLS is the fastest method even if number of iterations is increased compared to the basic WLS. FDWLS solution changes slightly but precision remains good.

SWLS1 does not converge at flat start $(\mathrm{k}=1)$, for $\mathrm{k}=2$ number of iterations and computation time is increased compared to the other methods. The solution is the same as Basic WLS.

SWLS2 applied at flat start $(\mathrm{k}=1)$ converges in half time compared to the basic WLS with a good quality of estimation even if number of iterations required is important compared to the other methods.

Table 1. Performance evaluation of WLS algorithms for IEEE 14 bus system under scenario1

\begin{tabular}{ccccc}
\hline Algorithm & Computation time (seconds) & Iterations number & MAPEV $(\%)$ & MAPEO $(\%)$ \\
\hline Basic WLS & 0.004541 & 4 & 1.28 & 2.41 \\
SWLS1 $(\mathrm{k}=1)$ & & Program does not converge & & 2.41 \\
SWLS1 $(\mathrm{k}=2)$ & 0.005751 & 7 & 1.28 & 2.41 \\
SWLS1 $(\mathrm{k}=3)$ & 0.004102 & 4 & 1.28 & 0.87 \\
SWLS2 $(\mathrm{k}=1)$ & 0.002113 & 8 & 0.57 & 2.5 \\
SWLS2 $(\mathrm{k}=2)$ & 0.002550 & 4 & 1.33 & 2.41 \\
SWLS2 $(\mathrm{k}=3)$ & 0.003545 & 4 & 0.34 & 0.45 \\
FDWLS & 0.001418 & 6 & \\
\hline
\end{tabular}

Figure 6 shows that FDWLS and SWLS2 $(\mathrm{k}=1)$ are closer to the true value than basic WLS, the same figure appearance was obtained for voltage angle. 


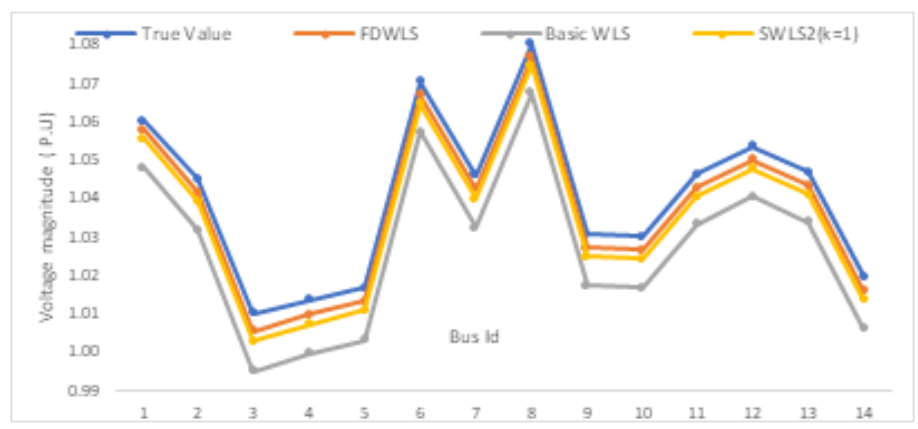

Figure 6. Estimated voltage magnitude (P.U) by different WLS algorithms for IEEE 14 bus system under scenario 1

\subsubsection{Simulation results for IEEE 118 bus system}

According to Table 2: here also, SWLS1 does not converge for $\mathrm{k}=1$. It seems that constant gain matrix associated to variable Jacobian may lead to convergence problems. FDWLS computation time is the lowest. The all methods have provided a good estimation quality.

Table 2. Performance evaluation of WLS algorithms for IEEE 118 bus system under scenario1

\begin{tabular}{|c|c|c|c|c|}
\hline Algorithm & Computation time (seconds) & Iterations number & MAPEV (\%) & МАРЕӨ $(\%)$ \\
\hline Basic WLS & 0.182347 & 4 & 1.79 & 1.74 \\
\hline SWLS1 $(k=1)$ & \multicolumn{4}{|c|}{ Program does not converge } \\
\hline SWLS1 (k=2) & 0.265629 & 7 & 1.79 & 1.74 \\
\hline SWLS1 (k=3) & 0.156127 & 4 & 1.79 & 1.74 \\
\hline SWLS2 (k=1) & 0.084726 & 10 & 1.81 & 1.13 \\
\hline SWLS2 (k=2) & 0.098518 & 5 & 1.77 & 1.72 \\
\hline SWLS2 (k=3) & 0.135495 & 4 & 1.79 & 1.74 \\
\hline FDWLS & 0.037558 & 5 & 2.07 & 1.1 \\
\hline
\end{tabular}

\subsubsection{Simulation results for 12 bus distribution system}

As noticed in Table 3, SWLS1 has converged for $\mathrm{k}=1$ because the final state is too close to the initial state (Basic WLS converges only in 3 iterations).

FDWLS convergence speed slowed down considerably compared to the other algorithms. The solution of FDWLS is the less accurate, and the program required a high iteration number (60,5 against 3 for the basic formulation). SWLS2 $(\mathrm{k}=1)$, converged in less time and its solution is accurate as the basic WLS ones.

Table 3. Performance evaluation of WLS algorithms for 12 bus distribution system under scenario1

\begin{tabular}{ccccc}
\hline Algorithm & Computation time (seconds) & Iterations number & MAPEV $(\%)$ & MAPEO $(\%)$ \\
\hline Basic WLS & 0.002201 & 3 & 0.01 & 1.02 \\
SWLS1 $(\mathrm{k}=1)$ & 0.003601 & 5 & 0.01 & 1.02 \\
SWLS1 $(\mathrm{k}=2)$ & 0.002021 & 3 & 0.01 & 1.02 \\
SWLS2 $(\mathrm{k}=1)$ & 0.001545 & 5 & 0.01 & 1.02 \\
SWLS2 $(\mathrm{k}=2)$ & 0.001906 & 3 & 0.01 & 1.02 \\
FDWLS & 0.003879 & 60.5 & 0.76 & 19.87 \\
\hline
\end{tabular}

Results are depicted in Figure 7: the differences between estimated voltages by SWLS2(k=1), Basic WLS and the true values are so small that they cannot be distinguished. 


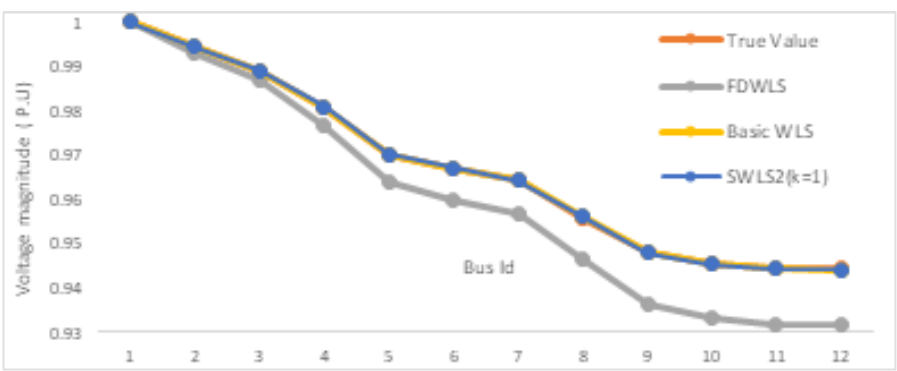

Figure 7. Estimated voltage magnitude (P.U) by different WLS algorithms for 12 bus distribution system under scenario1

\subsection{Results of scenario 2 (erroneous measurements)}

In this section, measurements were altered by a noise of $10 \%$ to evaluate the response of the studied algorithms to erroneous measurements.

\subsubsection{Simulation results for IEEE 14 bus system}

Table 4 shows that bad measurements alter the estimation quality for all WLS algorithms. Specially, FDWLS which provided the less accurate solution. However, FDWLS method still constantly the fastest one. For this case, SWLS1 have failed not only at flat start but also for the second iteration $(k=2)$.

According to Figure 8, All WLS algorithms solutions are far from the true values. The FDWLS curve is the furthest while Basic WLS curve is the nearest one to the true values curve. Those results are the inverse of those obtained in scenario 1.

Table 4. Performance evaluation of WLS Algorithms for IEEE 14 bus system under scenario2

\begin{tabular}{|c|c|c|c|c|}
\hline Algorithm & Computation time (seconds) & Iterations number & MAPEV (\%) & MAPEO (\%) \\
\hline Basic WLS & 0.005858 & 5 & 7.82 & 5.07 \\
\hline SWLS1 $(\mathrm{k}=1)$ & \multirow{2}{*}{\multicolumn{4}{|c|}{ Program does not converge }} \\
\hline SWLS1 $(\mathrm{k}=2)$ & & & & \\
\hline SWLS1 $(\mathrm{k}=3)$ & 0.005266 & 5 & 7.82 & 5.07 \\
\hline $\operatorname{SWLS2}(\mathrm{k}=1)$ & 0.002391 & 8 & 9.08 & 7.32 \\
\hline $\operatorname{SWLS2}(\mathrm{k}=2)$ & 0.003127 & 5 & 7.3 & 4.17 \\
\hline SWLS2 (k=3) & 0.003545 & 4 & 7.8 & 5.04 \\
\hline FDWLS & 0.001459 & 6.5 & 9.6 & 8.15 \\
\hline
\end{tabular}

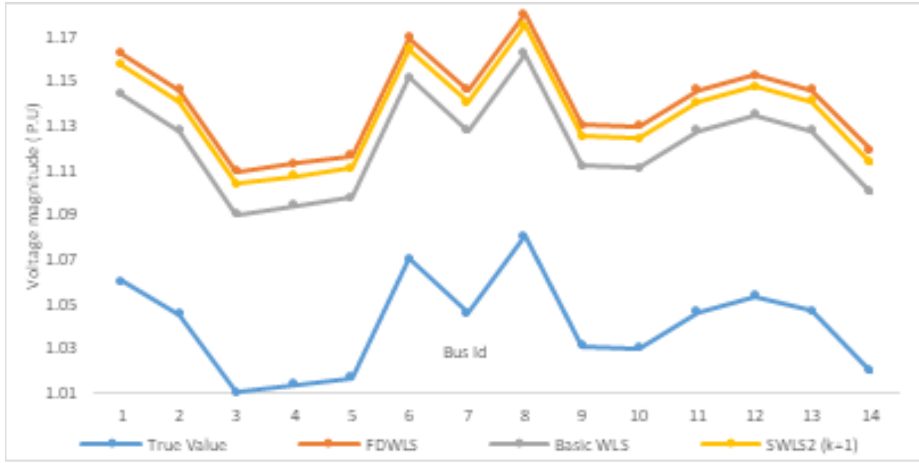

Figure 8. Estimated voltage magnitude (p.u) by different WLS algorithms for IEEE 14 bus system under scenario2

\subsubsection{Simulation results for IEEE 118 bus system}

Results presented in Table 5, are less affected by noise compared to the previous case IEEE 14 bus system, especially for voltage angle state. In fact, the set of measurements chosen for this studied model, presents a high redundancy level $(\eta=3)$ which allowed to the WLS Algorithms to provide good estimation. SWLS1 has converged for $\mathrm{k}=2$ in more iterations and computing time compared to the other algorithms.

Full versus decoupled constant matrices to speed up power system state estimation (Meriem Majdoub) 
Table 5. Performance Evaluation of WLS Algorithms for IEEE 118 Bus system under Scenario2

\begin{tabular}{ccccc}
\hline Algorithm & Computation time (seconds) & Iterations number & MAPEV $(\%)$ & MAPEӨ $(\%)$ \\
\hline Basic WLS & 0.184747 & 4 & 6.65 & 0.99 \\
SWLS1 $(\mathrm{k}=1)$ & & Program does not converge & & 0.99 \\
SWLS1 $(\mathrm{k}=2)$ & 0.456256 & 14 & 6.66 & 0.99 \\
SWLS1 $(\mathrm{k}=3)$ & 0.170388 & 4 & 6.65 & 1.85 \\
SWLS2 $(\mathrm{k}=1)$ & 0.084137 & 10 & 7.27 & 0.87 \\
SWLS2 $(\mathrm{k}=2)$ & 0.095147 & 5 & 6.54 & 0.99 \\
SWLS2 (k=3) & 0.130684 & 4 & 6.66 & 2.93 \\
FDWLS & 0.041343 & 5.5 & 8.37 & \\
\hline
\end{tabular}

\subsubsection{Simulation results for 12 bus distribution system}

As noticed in Table 6, here also the FDWLS has converged slowly with high iteration numbers. While, SWLS2(k=1), converges in reduced time compared to the basic WLS, with the same reliability.

Table 6. Performance evaluation of WLS algorithms for 12 bus distribution system under scenario2

\begin{tabular}{ccccc}
\hline Algorithm & Computation time (seconds) & Iterations number & MAPEV $(\%)$ & MAPEӨ (\%) \\
\hline Basic WLS & 0.002934 & 4 & 10.35 & 9.07 \\
SWLS1 (k=1) & 0.005536 & 9 & 10.35 & 9.06 \\
SWLS1 (k=2) & 0.002801 & 4 & 10.35 & 9.07 \\
SWLS2 (k=1) & 0.001602 & 6 & 10.34 & 9.08 \\
SWLS2 (k=2) & 0.002208 & 4 & 10.35 & 9.07 \\
FDWLS & 0.004408 & 68.5 & 9.6 & 9.11 \\
\hline
\end{tabular}

\section{DISCUSSION}

Applied on transmission systems with low R/X ratio, FDWLS is the fastest WLS Algorithm. It is 25 times faster than basic WLS even if convergence requires half to two iterations more than basic WLS (simplifications assumed diminish the true quadratic convergence properties of basic WLS). FDWLS requires half storage capacity since off diagonal elements of $\mathrm{H}$ and $\mathrm{G}$ matrices are neglected. Diagonal Jacobian elements are simples and contain only reactance branches. However, applied on distribution system with high R/X ratio, FDWLS was the slowest algorithm and required a huge iterations number because assumptions considered are based on transmission system features (active/reactive decoupling).

SWLS1 algorithm does not converge for first iterations. It seems that constant gain matrix associated to variable Jacobian may lead to convergence problems. SWLS1 is not reliable and does not present any advantages on reducing computation time.

SWLS2 algorithm applied at first iteration $(\mathrm{k}=1)$, requires the evaluation of gain and Jacobian matrices just once which reduces computation time. Indeed, SWLS2 $(\mathrm{k}=1)$ is 2 times faster than basic WLS even it requires a higher iterations number. SWLS2 $(\mathrm{k}=1)$ has the same characteristics of basic WLS which make it less sensitive to erroneous measurements and high R/X ratio compared to FDWLS and it could be applied in distribution network state estimation.

\section{CONCLUSION}

This paper has presented a performance comparison between two solutions to reduce computational burden of traditional Weighted Least Squares (WLS) Algorithm: Solution 1 based on full constant matrices (simplified methods SWLS1 / SWLS2) and Solution 2 based on decoupled constant matrices (Fast Decoupled WLS FDWLS). Algorithms have been tested on three study cases IEEE 14, 118 bus systems and a 12-bus rural distribution system. For each case, simulations were performed on accurate and erroneous measurements.

Simulation results have shown that the performance of FDWLS algorithm depends on the application environment: applied on transmission systems, FDWLS is the fastest WLS Algorithm. However, on distribution system with high R/X ratio, FDWLS was the slowest algorithm and required a huge iterations number. Therfore, FDWLS is not suitable for distribution system state estimation.

SWLS2 $(\mathrm{k}=1)$ is 2 times faster than basic WLS with the same reliability, which make it less sensitive to erroneous measurements and high R/X ratio compared to FDWLS. Therefore, SWLS2 applied at the flat start presents a good alternative to reduce computation time in future distribution system state estimation. 


\section{REFERENCES}

[1] Dejun Meng, Yong Wang, Xiuxia Tian, Liangliang Huang, Zongshuai Hu, Rusen Fan, "Least squares class power system state estimation algorithm efficiency analysis", Third International Conference on Technological Advances in Electrical, Electronics and Computer Engineering TAEECE, 2015.

[2] A. Monticelli, "State estimation in electric power systems a generalized approach", Springer, 2012.

[3] J.J. Allemong, L. Radu, A.M. Sasson, "A fast and reliable state estimation algorithm for aep's new control center," IEEE Transactions on Power Apparatus and Systems, vol. PAS-101, no. 4, Apr 1982.

[4] Ali Abur and Antonio Gomez Exposito, "Power system estimation: Theory and implementation", Marcel Dekker, Inc., 2004.

[5] A. Thabet, S. Chniba, D. Gaetan, M. Boutayeb, M.N. Abdelkrim, "Power systems load flow and state estimation: Modified methods and evaluation of stability and speeds computing", International Review of Electrical Engineering I.R.E.E, vol.5, no.3, May-Jun 2010.

[6] SURESH, Vishnu Sidaarth, "Comparison of solvers performance for load flow analysis," Transactions on Environment and Electrical Engineering, vol. 3, no. 1, pp. 26-32, Feb. 2019.

[7] Karimi, M., Shahriari, A., Aghamohammadi, M. R., Marzooghi, H., Terzija, V. "Application of Newton-based load flow methods for determining steady-state condition of well and ill-conditioned power systems: A review," International Journal of Electrical Power \& Energy Systems, vol. 113, pp. 298-309, Dec 2019.

[8] Tostado, M., Kamel, S., \& Jurado, F., "Several robust and efficient load flow techniques based on combined approach for ill-conditioned power systems," International Journal of Electrical Power \& Energy Systems, vol. 110, pp. 349-356, Sep 2019.

[9] Ailson P. De Moura, Adriano Aron F. De Moura, "Newton-Raphson power flow with constant matrices: A comparison with decoupled power flow methods," International Journal of Electrical Power \& Energy Systems, vol. 46, pp. 108-114, Mar 2013.

[10] Stanko Janković, Bojan Ivanović, "Application of combined Newton-Raphson method to large load flow model," Electric Power Systems Research, vol. 127, pp. 134-140, Oct 2015.

[11] Leandro Ramos de Araujo, Débora Rosana Ribeiro Penido, Nélio Alves do Amaral Filho, Tatianna Aparecida Pereira Beneteli, "Sensitivity analysis of convergence characteristics in power flow methods for distribution systems", Electrical Power and Energy Systems, vol. 97, pp. 211-219, Apr 2018.

[12] Md. Ashfaqur Rahman, Ganesh Kumar Venayagamoorthy, "Convergence of the fast state estimation for power systems," SAIEE Africa Research Journal, vol.108, no. 3, Sep 2017.

[13] Meriem Majdoub, Jamal Boukherouaa, Bouchra Cheddadi, Abdelaziz Belfqih, Omar Sabri, Touria Haidi, "Performance evaluation of two simplified algorithms of WLS power system state estimation," Przeglad Elektrotechniczny, vol, 1, no 12, 2018.

[14] Meriem Majdoub, Jamal Boukherouaa, Bouchra Cheddadi, Abdelaziz Belfqih, Omar Sabri, Touria Haidi, "Fast power system state estimation based on weighted least squares algorithm with constant matrices", SCA2018, Tetouan, Morocco, Oct 10-11 2018.

[15] Meriem Majdoub, Jamal Boukherouaa, Bouchra Cheddadi, Abdelaziz Belfqih, Omar Sabri, Touria Haidi, "Efficiency analysis of WLS algorithm with constant matrices on well and ill conditioned power systems", In: Ben Ahmed M., Boudhir A., Younes A. (eds) Innovations in Smart Cities Applications Edition 2. SCA 2018. Lecture Notes in Intelligent Transportation and Infrastructure. Springer, Cham, 2018.

[16] Majdoub Meriem, Cheddadi Bouchra, Belfqih Abdelaziz, Boukherouaa Jamal, Sabri Omar, El Mariami Faissal, Cherkaoui Nazha, "Study of state estimation using weighted-least-squares method", International Conference on Electrical Sciences and Technologies in Maghreb (CISTEM), 2016.

[17] Majdoub Meriem, Sabri Omar, Cheddadi Bouchra, Belfqih Abdelaziz, Boukherouaa Jamal, El Mariami Faissal, Cherkaoui Nazha, "Study of state estimation using weighted least squares method", International Journal of Advanced Engineering Research and Science (IJAERS), vol 3, Issue-8, Aug 2016.

[18] Sabri Omar, Majdoub Meriem, Cheddadi Bouchra, Belfqih Abdelaziz, Boukherouaa Jamal, El Mariami Faissal, Cherkaoui Nazha, "Correlation between weight, error and type of measurements in WLS State estimation of a Real Network", Przeglad Elektrotechniczny, ISSN 0033-2097, R. 93 NR 7/2017.

[19] Ankur Majumdar, "Security in power system state estimation", Ph.D. dissertation. Dept. Electrical and Electronic Engineering, Imperial College London, May 2016.

[20] K. S. Sastry Musti, Balasubrahmanyam P.V., Rajesh Reddy Malireddy, "Power system state estimation using Microsoft excel," Spreadsheets in Education (eJSiE), vol. 8, no. 1, Article 1, 2014.

[21] Mehdi Davoudi, Elssodani Abdelhadi Muhammed, "PMU placement to reduce state estimation bias considering parameters uncertainty," The 9th Power Systems Protection \& Control Conference (PSPC2015), Jan 2015.

[22] Power systems test case archive, Univ. Washington, Seattle, WA, USA, [Online] Available: https://www2.ee.washington.edu/research/pstca/, 1993.

[23] Jiaxiong Chen and Yuan Liao, "State Estimation and Power Flow Analysis of Power Systems", Journal of computers, vol 7, no.3, Mar 2012.

[24] Min Liu, "State estimation in a smart distribution system," Hkie Transactions, vol. 24, no. 1, pp. 1-8, 2017.

[25] D. Das, H.S. Nagi, D.P. Kothari, "Novel method for solving radial distribution networks", IEE Proc.-Gener. Transm. Distrib., vol. 141, no. 4, Jul 1994. 